\title{
Differential Sympathetic Neural Control of Oxygenation in Resting and Exercising Human Skeletal Muscle
}

\author{
Jim Hansen, Gail D. Thomas, Shannon A. Harris, William J. Parsons, and Ronald G. Victor \\ Department of Internal Medicine, Cardiology Division, Molecular Cardiology Laboratories, University of Texas Southwestern Medical \\ Center, Dallas, Texas 75235-8573
}

\begin{abstract}
Metabolic products of skeletal muscle contraction activate metaboreceptor muscle afferents that reflexively increase sympathetic nerve activity (SNA) targeted to both resting and exercising skeletal muscle. To determine effects of the increased sympathetic vasoconstrictor drive on muscle oxygenation, we measured changes in tissue oxygen stores and mitochondrial cytochrome $a, a_{3}$ redox state in rhythmically contracting human forearm muscles with near infrared spectroscopy while simultaneously measuring muscle SNA with microelectrodes. The major new finding is that the ability of reflex-sympathetic activation to decrease muscle oxygenation is abolished when the muscle is exercised at an intensity $>10 \%$ of maximal voluntary contraction (MVC). During high intensity handgrip (45\% MVC), contractioninduced decreases in muscle oxygenation remained stable despite progressive metaboreceptor-mediated reflex increases in SNA. During mild to moderate handgrips (20-33\% MVC) that do not evoke reflex-sympathetic activation, experimentally induced increases in muscle SNA had no effect on oxygenation in exercising muscles but produced robust decreases in oxygenation in resting muscles. The latter decreases were evident even during maximal metabolic vasodilation accompanying reactive hyperemia.

We conclude that in humans sympathetic neural control of skeletal muscle oxygenation is sensitive to modulation by metabolic events in the contracting muscles. These events are different from those involved in either metaboreceptor muscle afferent activation or reactive hyperemia. (J. Clin. Invest. 1996. 98:584-596.) Key words: sympathetic nervous system - microelectrodes - muscle contraction • vasoconstriction • tissue oxygenation
\end{abstract}

\section{Introduction}

The sympathetic nervous system produces many of the cardiovascular adjustments during exercise, including reflex increases in blood pressure, heart rate, and regional vascular re-

\footnotetext{
Address correspondence to Ronald G. Victor, Department of Internal Medicine Molecular Cardiology Laboratories, UT Southwestern Medical Center, 5323 Harry Hines Blvd., NB 11.200, Dallas, TX 75235-8573. Phone: 214-648-1400; FAX: 214-648-1450; E-mail: victor@ryburn.swmed.edu

Received for publication 19 October 1995 and accepted in revised form 3 May 1996.
}

J. Clin. Invest.

(C) The American Society for Clinical Investigation, Inc.

0021-9738/96/07/0584/13 \$2.00

Volume 98, Number 2, July 1996, 584-596 sistance. Although these adjustments match oxygen delivery to the metabolic demands of exercising skeletal muscle, the underlying mechanisms by which this matching occurs remain poorly understood.

As early as 1841, A.W. Volkmann (1) proposed that the accumulation of chemical products of contraction in skeletal muscle somehow signals the brain of a mismatch between muscle blood flow and metabolism and evokes compensatory neurocirculatory responses to minimize this perfusion mismatch. There now is unequivocal evidence that metabolic products of contraction (such as $\mathrm{H}^{+}$) activate chemically sensitive skeletal muscle afferents that reflexively increase efferent-sympathetic vasoconstrictor discharge (2-15). This reflex mechanism (termed the "muscle metaboreflex") has been shown to trigger parallel sympathetic activation in resting and exercising human skeletal muscle (16), but the resultant effect of this reflex-sympathetic activation on muscle blood flow and oxygenation remains poorly understood.

In quiescent skeletal muscle, reflex-sympathetic activation produces vasoconstriction (10), which is thought to optimize blood flow to metabolically active muscles. However, the functional consequence of reflex-sympathetic activation in contracting skeletal muscle has been the subject of considerable debate. Previous studies in intact animals and humans have suggested that sympathetic vasoconstriction in contracting muscle is both (a) well preserved (17-28), thereby partially offsetting metabolic vasodilation to maintain blood pressure (29); and (b) largely negated by metabolic vasodilation (30-38), thereby optimizing muscle perfusion. The latter concept, initially termed "functional sympatholysis" (30) recently has been extended by reductionist microcirculatory preparations (39-45) demonstrating that certain local metabolic consequences of contraction (e.g., intramuscular acidosis, hypoxia) interfere with specific signal transduction pathways mediating alpha-adrenergic vasoconstriction. Furthermore, the alpha adrenergic receptors that are most susceptible to such metabolic inhibition are those located on the distal nutrient arterioles, which are most accessible to metabolic products of contraction; in contrast, adrenergic receptors located on more proximal resistance arteries are not so accessible to these metabolic products (42). Our recent studies (33) further extend this concept by implicating a key role for intramuscular acidosis, since sympathetically mediated vasoconstriction in rat hindlimb muscle was greatly attenuated during intense contraction of highly glycolytic, but not of oxidative, muscles.

Taken together, these recent animal studies led us to hypothesize that contraction-induced metabolic inhibition of sympathetic vasoconstriction in mainly nutrient arterioles negates an otherwise deleterious effect of reflex-sympathetic activation on muscle oxygenation. The ability to continuously measure changes in tissue oxygen stores and mitochondrial oxygen availability in contracting skeletal muscle with near infrared spectroscopy, while simultaneously measuring muscle sympa- 
thetic nerve activity (SNA) ${ }^{1}$ with intraneural microelectrodes, provided a new opportunity to test this hypothesis in humans. Specifically, we asked: $(a)$ do reflex increases in muscle SNA lead to decreased oxygenation in resting, but not in contracting skeletal muscle; and $(b)$ is such functional sympatholysis evident mainly during intense rather than mild muscle contraction? We postulated that the minimum level of contraction that elicits functional sympatholysis is the same high intensity that is needed to elicit metaboreflex-mediated increases in muscle SNA. This would implicate a common metabolic mechanism, such as glycolytic production of $\mathrm{H}^{+}$, in the coordinate regulation of reflex-sympathetic activation and mitochondrial oxygenation in human skeletal muscle.

\section{Methods}

The study protocols were approved by the Institutional Review Board at University of Texas Southwestern Medical Center, and written informed consent was obtained from each subject prior to the study. We studied a total of 29 healthy volunteer subjects, 21 male and 8 female, 19 to 47 years of age (mean age of 25). Several of the subjects participated in more than one protocol on separate occasions.

\section{General methods}

Subjects were studied in the supine position. Heart rate was measured continuously by electrocardiography and arterial pressure by finger photoplethysmography (Finapres; Ohmeda, Englewood, CO). Respiratory excursions were monitored with a strain-gauge pneumograph; during the experimental protocols, subjects were instructed to avoid performance of a Valsalva maneuver or prolonged expiration because these respiratory maneuvers can stimulate sympathetic nerve activity (46). All measurements were recorded continuously on an FM tape recorder (R-71; TEAC, Japan) and on an electrostatic recorder (ES 1000; Gould Inc., Cleveland, OH).

\section{Recording of sympathetic nerve discharge}

Multiunit recordings of postganglionic SNA targeted to the skeletal muscle bed were obtained with unipolar tungsten microelectrodes inserted selectively into muscle nerve fascicles of the peroneal nerve posterior to the fibular head by microneurography (47). Briefly, the neural signals were amplified (by 20-50 $\times 10^{3}$ ), filtered (bandwidth of 700 to $2,000 \mathrm{~Hz}$ ), rectified, and integrated (time constant of $0.1 \mathrm{~s}$ ) to obtain a mean voltage display of sympathetic activity. A recording of muscle SNA was considered acceptable when the neurograms revealed spontaneous, pulse-synchronous bursts of neural activity, with a minimum signal-to-noise ratio of 3:1, that increased during phases II and III of the Valsalva maneuver but not during arousal stimuli (loud noise, skin pinch).

Sympathetic bursts were detected by inspection of the filtered and mean voltage neurograms; the interobserver and intraobserver variability in identifying bursts is $<10$ and $<5 \%$, respectively (6). Inadvertent contraction of the leg muscles adjacent to the recording electrode produces electromyographic artifacts that are easily distinguished from sympathetic bursts; neurograms that revealed such artifacts were excluded from analysis. SNA was expressed as $(a)$ the number of bursts of sympathetic activity per minute, and $(b)$ the number of bursts per minute multiplied by the mean burst amplitude in that minute (total activity).

\section{In vivo near infrared multiwavelength spectroscopy}

Near infrared spectroscopy was performed according to the method

1. Abbreviations used in this paper: cyt $a, a_{3}$, cytochrome $a, a_{3} ; \mathrm{LBNP}$, lower body negative pressure; MVC, maximal voluntary contraction; SNA, sympathetic nerve activity; TLS, total labile signal. of Piantadosi and co-workers (48-50). Detailed descriptions of near infrared spectroscopy applied to skeletal muscle, cardiac muscle, and brain have been published previously (e.g., references 51-56) and the theoretical background and in vivo validation studies have been reviewed (48-50, 57-61).

Briefly, the near infrared method exploits the principle that laser light with wavelengths in the 700-900-nm range penetrates tissues with relative ease, and is absorbed by the copper moiety $\left(\mathrm{Cu}_{\mathrm{A}}\right)$ in cytochrome $a, a_{3}$ and by the iron-porphyrin moieties in oxygenated and deoxygenated hemoglobin and myoglobin. Changes in absorption are proportional to changes in the relative concentrations of oxidized cytochrome $a, a_{3}$, oxygenated hemoglobin and myoglobin, and deoxygenated hemoglobin and myoglobin. Thus, the technique provides continuous measurement of several indices of the adequacy of muscle oxygen delivery relative to use at the intracellular and microcirculatory level. These include:

Redox state of cytochrome $a, a_{3}$. Cytochrome $a, a_{3}$ is the terminal member of the electron transport chain and is located on the inner mitochondrial membrane where it catalyzes the transfer of electrons to molecular oxygen, thereby making free energy available for the synthesis of ATP via oxidative phosphorylation. The final step in mitochondrial respiration is a four-electron transfer between cytochrome $a, a_{3}$ and oxygen. In view of the high affinity constant, rapid rate, and compartmental properties of the reaction between cytochrome $a, a_{3}$ and molecular $\mathrm{O}_{2}$, the redox state of cytochrome $a, a_{3}$ is a sensitive indicator of relative changes in mitochondrial oxygen availability. Thus, a decrease in cytochrome $a, a_{3}$ (cyt $\left.a, a_{3}\right)$ redox state suggests diminished mitochondrial $\mathrm{O}_{2}$ availability relative to the rate of electron transport, which is determined by metabolic demand.

Tissue oxygen stores. The near infrared method also permits measurement of changes in the absorption by iron-porphyrin moieties in oxygenated hemoglobin plus myoglobin $\left(\mathrm{tHbO}_{2}+\mathrm{MbO}_{2}\right.$, or "tissue oxygen stores"), and deoxygenated hemoglobin plus myoglobin $(\mathrm{tHb}+\mathrm{Mb})$. It is not possible to estimate the fraction of $\mathrm{O}_{2}$ in tissue hemoglobin vs myoglobin, since the absorption spectra of oxyhemoglobin and oxymyoglobin (and the spectra of deoxyhemoglobin and deoxymyoglobin) are nearly identical. Importantly, the oxygendependent changes in the hemoglobin absorption reflect changes occurring mainly in the microvessels, rather than in the resistance vessels. Due to the high extinction coefficients of whole blood, vessels greater than $1 \mathrm{~mm}$ in diameter must be considered maximal absorbers of photons. Thus, photons that successfully migrate through muscle will primarily proceed through minimal absorbers, such as small arterioles, capillaries, and venules (61). Furthermore, since most of the hemoglobin is located in capacitance vessels, the hemoglobin component of the optical signals is most sensitive to changes in capillary and venous $\mathrm{O}_{2}$ content. In the present study, similar directional changes in the cytochrome $a, a_{3}$ redox state and $\mathrm{tHbO}_{2}+\mathrm{MbO}_{2}$, and reciprocal changes in $\mathrm{tHb}+\mathrm{Mb}$ reflect changes in muscle oxygenation.

In the present experiments, near infrared signals were obtained by means of two fiber optical bundles (optrodes), which were placed over the region of the flexor digitorum profundus muscle of the left arm, which is the main active muscle mass recruited during the type of handgrip performed (62). The optrodes were applied to the skin oriented parallel to each other $\sim 2.3 \mathrm{~cm}$ apart for optimal reflectance measurements. The average depth of penetration of the near infrared light is a function of the distance between the optrodes (57). The photons traverse a banana-shaped region underlying the two optrodes with the average depth of penetration equaling half the distance between the optrodes $(\sim 1.2 \mathrm{~cm})$. In preliminary experiments, we found that rhythmic handgrip can cause two types of motion artifacts, which are readily detectable and preventable: $(a)$ complete loss of contact between optrodes and skin, which leads to an obvious loss of all optical signals, and $(b)$ inadvertent movement of the optrodes from their optimal placement, but without complete displacement from the skin. This latter motion artifact is readily detectable by $(a)$ abrupt changes in all optical signals and $(b)$ failure of the signals to return to the pre- 
exercise baseline values during recovery. Such motion artifacts were avoided with the use of a spring loaded stereotactic instrument that effectively maintained the stable position of the optrodes during rhythmic handgrip. In each experiment, we documented absence of a baseline shift by ensuring that during recovery all optical signals returned to within $5 \%$ of the total labile signal (TLS) (54). All near infrared measurements (i.e., cyt $a, a_{3}, \mathrm{tHbO}_{2}+\mathrm{MbO}_{2}$ and $\mathrm{tHb}+\mathrm{Mb}$ ) were obtained at a sampling rate of $1 \mathrm{~Hz}$, and each data point displayed as the mathematical average of 10 consecutive measurements. The maximal change in the near infrared signals was determined in each experiment as the difference between baseline and complete deoxygenation accomplished with circulatory arrest (pneumatic cuff at $280 \mathrm{mmHg}$ at the level of the brachial artery for $8 \mathrm{~min}$ in resting muscle or for $2 \mathrm{~min}$ after handgrip exercise). The magnitude of this difference defines the TLS for each parameter, and all changes are expressed as a percentage of the total labile signal (52). The reproducibility of changes in optical density to a given intervention in a given subject was determined in preliminary studies where handgrip at $45 \%$ maximal voluntary contraction (MVC), lower body negative pressure (LBNP) at $-20 \mathrm{mmHg}$, and forearm circulatory arrest (to obtain the TLS) was performed twice on four subjects. The differences (mean \pm SD) in the change in optical density between the two tests were (cyt $a, a_{3}$, $\mathrm{tHbO}_{2}+\mathrm{MbO}_{2}, \mathrm{tHb}+\mathrm{Mb}$ ): HG: $-0.02 \pm 0.04,0.01 \pm 0.06$, and $0.05 \pm$ 0.02 . TLS: $-0.01 \pm 0.04,-0.01 \pm 0.04$, and $0.01 \pm 0.03$. LBNP: $-0.02 \pm$ $0.02,-0.01 \pm 0.01$, and $-0.01 \pm 0.02$. This corresponds to coefficients of variability (SD of the difference/mean change with intervention) of optical density changes between 4 and $16 \%$.

The near infrared spectrophotometer, which was constructed in Dr. Claude Piantadosi's laboratory (53-55), consists of four GaAIAs laser diodes and circuitry for photodetection, demodulation, and logarithmic amplification of the optical signals. The four lasers in sequence provide pulses of monochromatic light (1.5-nm bandwidth) at $775,810,870$, and $904 \mathrm{~nm}$. One fiber-optic bundle was used for laser pulse transmission and monitoring of incident light by a reference photomultiplier tube, and a second fiber-optic bundle was used to receive photons from the tissue region of illumination, for detection by a second photomultiplier tube. Photocurrents were integrated, demultiplexed, and fed through a log-ratio amplifier. These voltages were then input to a microprocessor and algorithms for the contribution of each absorber at each wavelength were used to solve for three unknowns: changes in cyt $a, a_{3}, \mathrm{tHbO}_{2}+\mathrm{MbO}_{2}$, and $\mathrm{tHb}+\mathrm{Mb}$. These metabolic signals were displayed on a computer screen in real time as changes in optical density $(\Delta \mathrm{OD})$.

Near infrared spectroscopy has been validated extensively (48-50, 59-61) and the algorithms used to separate cyt $a, a_{3}, \mathrm{tHbO}_{2}+\mathrm{MbO}_{2}$, and $\mathrm{tHb}+\mathrm{Mb}$ signals have been published $(54,55,60)$. The validity of these algorithms has been established in experimental animal models using exchange transfusions with perfluorocarbon blood substitutes (60). The seminal observation is that after five exchanges, cyt $a, a_{3}$ from rat brain remains stable (decreases by $<4 \%$ ), despite a $75 \%$ reduction in $\mathrm{tHbO}_{2}+\mathrm{MbO}_{2}$ (60). Furthermore, supporting evidence has been obtained using venous occlusion in the human forearm, a technique that does not alter arterial inflow of oxygenated blood such that the $\mathrm{tHbO}_{2}+\mathrm{MbO}_{2}$ signal remains unchanged, but which redistributes the oxygenated blood away from the nutrient circulation, resulting in a $58 \%$ decrease in cyt $a, a_{3}(59)$. The main limitations of near infrared spectroscopy currently are $(a)$ the difficulty in assessing the precise degree of separation between the cyt $a, a_{3}$ and the $\mathrm{tHbO}_{2}+$ $\mathrm{MbO}_{2}$ and $\mathrm{tHb}+\mathrm{Mb}$ signals; and $(b)$ the optical responses reflect relative rather than absolute changes in the concentrations of the absorbers, because the exact pathlength of transmitted photons is unknown (50). These limitations, however, are not relevant to the interpretation of the present experiments, where all the optical indices of muscle oxygenation were used to indicate changes in oxygen delivery relative to metabolic demand.

\section{Venous occlusion plethysmography}

Forearm blood flow during LBNP was measured with venous occlu- sion plethysmography according to the technique of Siggaard-Andersen (63). An air-filled latex cuff was placed $\sim 5 \mathrm{~cm}$ distal to the antecubital fossa and the arm was elevated to produce venous drainage. Circulation to the hand was arrested by inflating a cuff on the wrist to $250 \mathrm{mmHg}$ during determinations of forearm flow. The pressure of the venous congesting cuff on the upper arm was $40 \mathrm{mmHg}$. Forearm blood flow was measured at 15 -s intervals and expressed in milliliters per minute per $100 \mathrm{ml}$ of forearm volume. Forearm vascular resistance was calculated as mean arterial pressure/forearm blood flow. Mean arterial pressure was calculated as diastolic pressure + (pulse pressure/3).

\section{Handgrip exercise}

Handgrip exercise was performed with a custom-made handgrip dynamometer connected to a force transducer (Interface; MFG, Scottsdale, AZ). Force output from the handgrip device was recorded on paper and displayed on an oscilloscope to provide the subject with visual feedback. Before the experiment, each subject's MVC was determined as the best of four to five trials with verbal encouragement to improve at each trial. The exercise protocols consisted of $5 \mathrm{~min}$ of intermittent isometric exercise where subjects matched force production to a visual target, to the rhythm of a metronome (40 beats/min) with a $50 \%$ duty cycle.

Rhythmic handgrip at $45 \%$ MVC is accompanied by increases in muscle SNA due to activation of the muscle metaboreflex (7-9). In contrast, rhythmic handgrip at $5,10,20$, or $33 \%$ MVC does not engage the muscle metaboreflex and does not increase muscle SNA (7-9).

\section{Lower body negative pressure}

The subject's lower body was enclosed in a negative pressure chamber to the level of the iliac crest. An opening was created on one side of the chamber to allow performance of the microneurographic technique for recording SNA from the peroneal nerve in the right leg. Once a stable recording of SNA was obtained, the opening was closed and sealed during the protocol. The pressure inside of the LBNP chamber was measured by a Statham transducer (Gould Inc., Oxnard, CA). LBNP at $-20 \mathrm{mmHg}$ was used to selectively unload the cardiopulmonary baroreceptors, thereby producing highly reproducible increases in muscle SNA, without concomitant changes in systemic arterial pressure $(64,65)$.

\section{Administration of pharmacologic agents}

In some studies, bretylium tosylate (Astra Scientific Intl., Westborough, MA) was administered to one forearm by a regional intravenous technique ("Bier-block") to selectively inhibit sympathetic neurotransmission in the forearm (66). An intravenous catheter was inserted into a forearm vein, the forearm was elevated above heart level and exsanguinated with a compressing bandage. A tourniquet cuff was inflated on the upper arm and kept at a constant pressure of $280 \mathrm{mmHg}$. Bretylium tosylate $(1 \mathrm{mg} / \mathrm{kg}$ body wt) was dissolved in 40 $\mathrm{ml}$ saline and instilled into the forearm, which was kept ischemic for $20 \mathrm{~min}$ after the bretylium administration. We determined in pilotstudies that this technique selectively abolishes sympathetic vasoconstriction in the experimental forearm, as evidenced by absence of the forearm vasoconstrictor response to LBNP, for at least $4 \mathrm{~h}$ after the procedure, but it has no effect on systemic blood pressure or on sympathetic vasoconstriction in the contralateral forearm.

\section{Specific protocols}

Protocol 1. Temporal relations between changes in muscle sympathetic nerve activity (SNA) and muscle oxygenation during high intensity rhythmic handgrip (10 experiments performed on 10 subjects). By examining closely the temporal relations between changes in muscle SNA and muscle oxygenation during handgrip at $45 \%$ MVC, we sought to determine if the characteristically slow and progressive time course of reflex-sympathetic activation in skeletal muscle during exercise is reflected in the time course of the decreases in oxygenation of exercising skeletal muscle. 
Blood pressure, heart rate, respiration, force output, muscle SNA (recorded from the peroneal nerve), and near infrared signals (from the left flexor digitorum profundus muscle) were recorded at baseline and during $5 \mathrm{~min}$ of rhythmic handgrip at $45 \%$ MVC performed with the left forearm. Immediately before the end of the exercise, a tourniquet cuff was inflated around the upper arm and kept at $280 \mathrm{mmHg}$ for $2 \mathrm{~min}$ to produce postexercise forearm ischemia. This maneuver rapidly elicits the maximal response in the near infrared signals and it sustains activation of the muscle metaboreflex by trapping the metabolic products of contraction in the forearm (6).

The near infrared signals were recorded from the exercising forearm muscles. However, because of the technical difficulty in recording SNA to exercising forearm muscles, SNA to resting leg muscle was used as an index of SNA to exercising forearm muscle. The validity of this approach is provided by our previous experiments in which we documented that metaboreflex activation triggers parallel increases in SNA to resting and exercising human skeletal muscle in the lower leg
(16), and the documentation that, during handgrip exercise, the muscle metaboreflex has been demonstrated to trigger parallel increases in SNA targeted to the skeletal muscle of both the arm and leg (67).

Protocol 2. Effects of reflex-sympathetic activation on muscle oxygenation in resting human skeletal muscle (4 experiments performed on 4 subjects). The aim of this protocol was to validate the assumption that, during nonhypotensive LBNP, decreases in the oxygenation of resting forearm muscle are mediated by reflex-sympathetic vasoconstriction.

Blood pressure, heart rate, near infrared signals, and forearm blood flow (plethysmography) were measured for 2 min each before, during, and after application of LBNP at $-20 \mathrm{mmHg}$. This level of LBNP reproducibly increases muscle SNA by selectively unloading mainly cardiopulmonary baroreceptors, without changing arterial blood pressure (i.e., muscle perfusion pressure) (65). These experiments were performed both before and $1 \mathrm{~h}$ after regional forearm sympathetic blockade with bretylium.
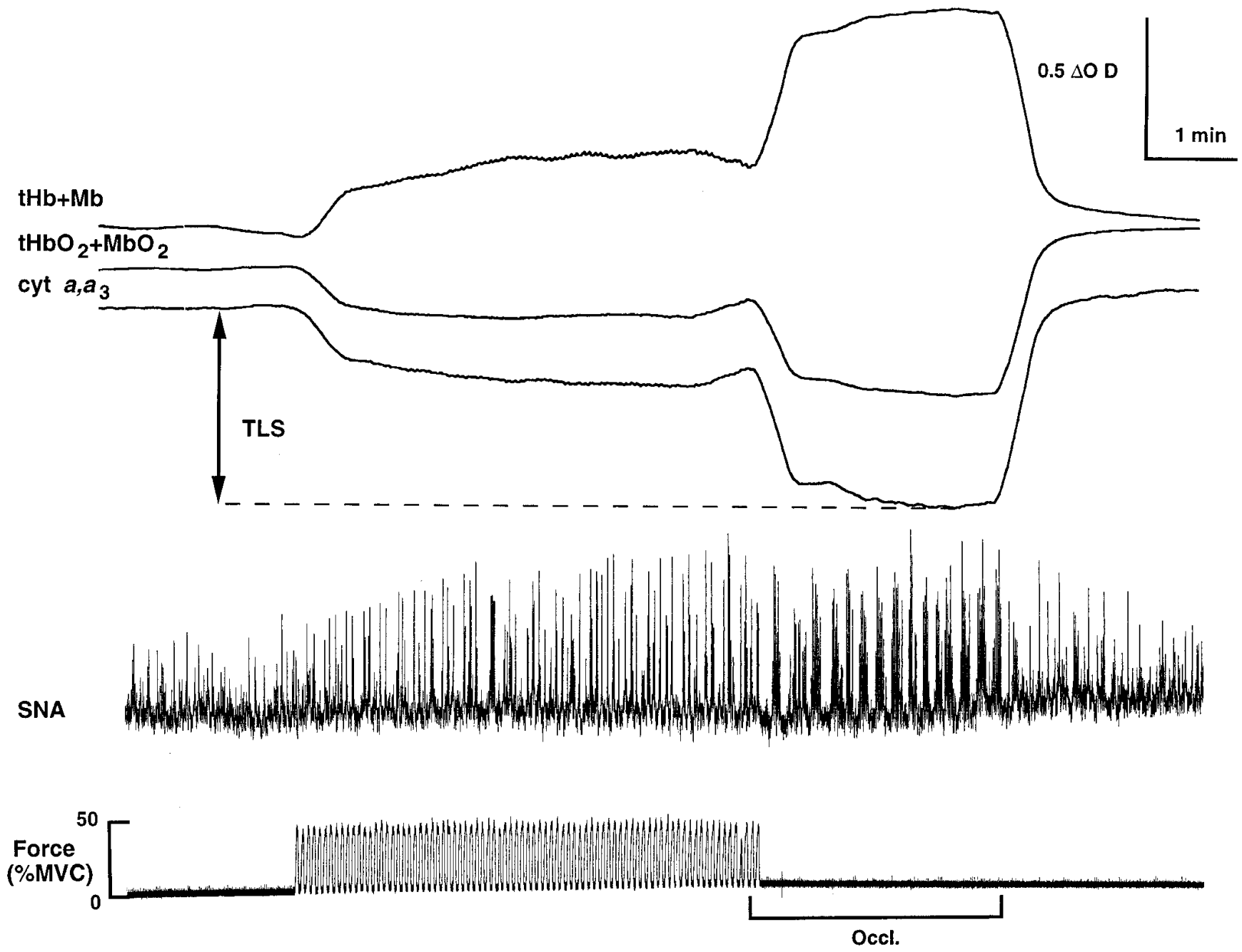

Figure 1. Segments of an original record showing simultaneous recordings of near infrared optical signals from forearm muscle (top), mean voltage neurogram of muscle-sympathetic nerve activity (center), and handgrip force (bottom). Continuous tracings are shown at rest, during 5 min of rhythmic handgrip at $45 \% \mathrm{MVC}, 2$ min of post-exercise forearm vascular occlusion, and recovery. In this subject, handgrip produced rapid decreases in cyt $a, a_{3}$ and in tissue oxygen stores $\left(\mathrm{tHbO}_{2}+\mathrm{MbO}_{2}\right)$, and a corresponding increase in deoxyhemoglobin and deoxymyoglobin $(\mathrm{tHb}+\mathrm{Mb})$. The majority of this change occurred within $30 \mathrm{~s}$ of the onset of exercise. In contrast, sympathetic nerve activity increased slowly and progressively, with the majority of the change occurring after the 1st min of exercise, demonstrating a clear temporal dissociation between the two responses. Complete forearm vascular occlusion $(\mathrm{Occl}$. $)$ after the exercise produced the peak deoxygenation that was used to determine the TLS, indicated by the arrow. Of note, the increase in muscle sympathetic nerve activity was maintained during occlusion, demonstrating activation of the muscle metaboreflex. 
Table I. Responses during Rhythmic Handgrip at 45\% Maximal Voluntary Contraction and Posthandgrip Forearm Vascular Occlusion

\begin{tabular}{|c|c|c|c|c|c|c|c|c|c|c|c|}
\hline & \multirow[b]{2}{*}{ Baseline } & \multicolumn{9}{|c|}{ Rhythmic handgrip, at $45 \%$ maximal voluntary contraction } & \multirow[b]{2}{*}{ Vascular occl.* } \\
\hline & & $30 \mathrm{~s}$ & $60 \mathrm{~s}$ & $90 \mathrm{~s}$ & $120 \mathrm{~s}$ & $150 \mathrm{~s}$ & $180 \mathrm{~s}$ & $210 \mathrm{~s}$ & $240 \mathrm{~s}$ & $270 \mathrm{~s}$ & \\
\hline $\begin{array}{l}\text { Mean arterial } \\
\text { pressure }(\mathrm{mmHg})\end{array}$ & $105 \pm 4$ & $112 \pm 4$ & $116 \pm 4 *$ & $119 \pm 5^{\ddagger}$ & $123 \pm 4^{\ddagger}$ & $126 \pm 5^{\ddagger}$ & $130 \pm 6^{\ddagger}$ & $132 \pm 6^{\ddagger}$ & $133 \pm 6^{\ddagger}$ & $132 \pm 6^{\ddagger}$ & $123 \pm 44^{\ddagger}$ \\
\hline \multicolumn{12}{|l|}{ Muscle SNA } \\
\hline Total activity (U) & $168 \pm 34$ & $211 \pm 57$ & $214 \pm 46$ & $271 \pm 46^{\ddagger}$ & $268 \pm 77$ & $290 \pm 61^{\ddagger}$ & $288 \pm 55^{+}$ & $324 \pm 58^{\ddagger}$ & $361 \pm 81^{\ddagger}$ & $351 \pm 59^{\ddagger}$ & $375 \pm 50^{\ddagger}$ \\
\hline $\begin{array}{l}\Delta \text { Total activity } \\
\text { (percentage) }\end{array}$ & 0 & $20 \pm 13$ & $31 \pm 11$ & $77 \pm 16$ & $57 \pm 18$ & $81 \pm 16$ & $87 \pm 19^{\ddagger}$ & $120 \pm 36^{\ddagger}$ & $136 \pm 42^{\ddagger}$ & $140 \pm 32^{\ddagger}$ & $169 \pm 52^{\ddagger}$ \\
\hline \multicolumn{12}{|l|}{$\begin{array}{l}\text { Near infrared } \\
\text { optical responses }\end{array}$} \\
\hline $\begin{array}{l}\Delta \text { cyt } a, a_{3} \\
\text { ( percentageTLS) }\end{array}$ & 0 & $-46 \pm 6^{\ddagger}$ & $-52 \pm 6^{\ddagger}$ & $-53 \pm 2^{\ddagger}$ & $-53 \pm 1^{\ddagger}$ & $-54 \pm 4^{\ddagger}$ & $-54 \pm 4^{\ddagger}$ & $-52 \pm 5^{\dot{z}}$ & $-52 \pm 5^{\ddagger}$ & $-54 \pm 4^{\ddagger}$ & $-100^{\ddagger}$ \\
\hline $\begin{array}{l}\Delta \mathrm{tHbO}_{2}+\mathrm{MbO}_{2} \\
\text { (percentage TLS) }\end{array}$ & 0 & $-50 \pm 6^{\ddagger}$ & $-54 \pm 6^{\ddagger}$ & $-52 \pm 5^{\ddagger}$ & $-51 \pm 4^{\ddagger}$ & $-51 \pm 5^{\ddagger}$ & $-50 \pm 4^{\ddagger}$ & $-50 \pm 4^{\ddagger}$ & $-47 \pm 4^{\ddagger}$ & $-47 \pm 4^{\ddagger}$ & $-100^{\ddagger}$ \\
\hline $\begin{array}{l}\Delta \mathrm{tHb}+\mathrm{Mb} \\
\text { (percentage TLS) }\end{array}$ & 0 & $41 \pm 7^{\ddagger}$ & $47 \pm 7^{\ddagger}$ & $50 \pm 6^{\ddagger}$ & $51 \pm 5^{\ddagger}$ & $53 \pm 6^{\ddagger}$ & $56 \pm 6^{\ddagger}$ & $55 \pm 7^{\ddagger}$ & $54 \pm 6^{\ddagger}$ & $56 \pm 6^{\ddagger}$ & $100^{\ddagger}$ \\
\hline
\end{tabular}

Data shown as mean \pm SEM for 10 subjects; *occl., occlusion; ${ }^{*}$ significant responses $(P<0.05$ vs baseline $)$.

Protocol 3. Effects of forearm sympathetic blockade on the changes in muscle oxygenation during high intensity rhythmic handgrip ( 4 experiments performed on 4 subjects). The aim of this protocol was to determine if pharmacologic blockade of sympathetic neurotransmission in the exercising arm would alter the magnitude or time course of muscle deoxygenation during handgrip. If the responses to handgrip were similar after sympathetic blockade, it would support our hypothesis that the effect of functional sympatholysis normally produced during handgrip equals the effect of chemical sympatholysis in these experiments.

Blood pressure, heart rate, force output, and near infrared signals (from the left flexor digitorum profundus muscle) were recorded at baseline and during 5 min of rhythmic handgrip at $45 \%$ MVC performed with the left forearm before and $1 \mathrm{~h}$ after forearm sympathetic blockade with bretylium. Each exercise bout was followed by 2 min of forearm circulatory arrest to establish the maximal decrease in the near infrared signals. The efficacy of sympathetic blockade was documented by loss of reflex deoxygenation in the forearm in response to $2 \mathrm{~min}$ of LBNP at -20 and $-40 \mathrm{mmHg}$.

Protocol 4. Effects of reflex-sympathetic activation on muscle oxygenation in exercising human skeletal muscle (16 experiments performed on 10 subjects). The aim of this protocol was to test our main hypothesis that the ability of reflex-sympathetic activation to elicit decreases in muscle oxygenation is attenuated when the muscle is exercised. To test this, reflex increases in muscle SNA evoked by a given level of LBNP $(-20 \mathrm{mmHg})$ were superimposed on levels of rhythmic handgrip exercise ( 20 or $33 \%$ MVC) that by themselves do not alter muscle SNA.

Blood pressure, heart rate, respiration, handgrip force, muscle SNA, and near infrared signals were recorded in response to LBNP $(-20 \mathrm{mmHg}$ for $2 \mathrm{~min}$ ) and rhythmic handgrip (20 or 33\% MVC for 5 min each) performed alone or in combination. The LBNP was applied during the $3 \mathrm{rd}$ and 4 th min of each 5-min exercise period. Each exercise bout was followed by two min of forearm circulatory arrest to establish the maximal decrease in the near infrared signals. All 10 subjects performed handgrip at $20 \% \mathrm{MVC}$ and in 6 of these subjects the experiments were repeated using a handgrip at $33 \% \mathrm{MVC}$, a level that causes greater muscle deoxygenation than handgrip at $20 \%$ MVC, but without producing reflex increases in muscle SNA.

Protocol 4a. Effects of mechanical vascular occlusion on muscle oxygenation in exercising human skeletal muscle ( 5 experiments per- formed on 5 subjects). The aim of this subprotocol was to compare the effects of reduced oxygen delivery on the time-dependent decreases in cyt $a, a_{3}$ and $\mathrm{tHbO}_{2}+\mathrm{MbO}_{2}$ at rest and during rhythmic handgrip. In preliminary experiments, we determined that $1 \mathrm{~min}$ of complete circulatory arrest (pneumatic cuff inflated to $280 \mathrm{mmHg}$ ) of a resting forearm produced decreases in muscle oxygenation equivalent to those produced by $2 \mathrm{~min}$ of LBNP at $-20 \mathrm{mmHg}$. The brief circulatory arrest was used as an internal control for decreases in oxygen delivery resulting from reflex-sympathetic vasoconstriction.

Blood pressure, heart rate, handgrip force, and near infrared signals were recorded in response to $1 \mathrm{~min}$ of complete forearm circulatory arrest at rest and during rhythmic handgrip (33\% MVC for $5 \mathrm{~min}$ ) performed alone or in combination. The forearm circulatory arrest was applied during the 3rd min of each 5-min exercise period. Each exercise bout was followed by $2 \mathrm{~min}$ of forearm circulatory arrest to establish the maximal decrease in the near infrared optical signals.

Protocol $4 b$. Effects of reflex-sympathetic activation on muscle oxygenation in resting forearm muscle during handgrip exercise with the contralateral arm (4 experiments performed on 4 subjects). In the above subprotocol, near infrared signals were recorded from the exercising forearm muscles. The aim of this subprotocol was to test the hypothesis that functional sympatholysis is localized to the exercising muscle and is not caused by some systemic effect of exercise. To accomplish this aim, near infrared signals were recorded from the resting left forearm during exercise of the contralateral right forearm.

Blood pressure, heart rate, force output, and near infrared signals from the left forearm were measured during LBNP at $-20 \mathrm{mmHg}$ and rhythmic handgrip at $33 \%$ MVC with the right arm performed alone and in combination.

Protocol 5. Effects of reflex-sympathetic activation on muscle oxygenation in exercising human forearm skeletal muscle during graded low intensity handgrip (12 experiments performed on 4 subjects). The aim of this protocol was to determine the minimum intensity of rhythmic handgrip at which functional sympatholysis could be detected, as evidenced by attenuation in the decrease in muscle oxygenation evoked by LBNP.

Blood pressure, heart rate, force output, and near infrared signals were measured in response to LBNP alone and superimposed during rhythmic handgrip performed as separate 5 -min bouts of 5, 10, or $20 \%$ MVC. The order was random with at least $15 \mathrm{~min}$ of recovery 
A

cyt $a, a_{3}$

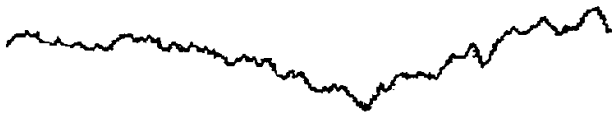

$\mathrm{tHbO}_{2}+\mathrm{MbO}_{2}$

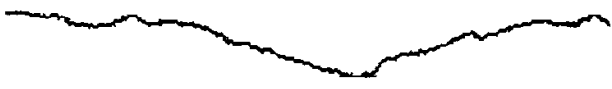

Forearm

Blood

$(\mathrm{ml} / \mathrm{min} / 100 \mathrm{ml}$

tissue)

Forearm

Vascular

Resistance

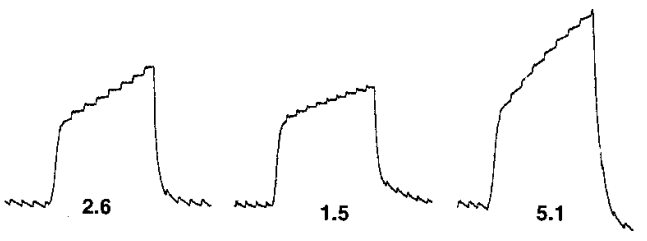

25.8

39.6

11.9

LBNP

B
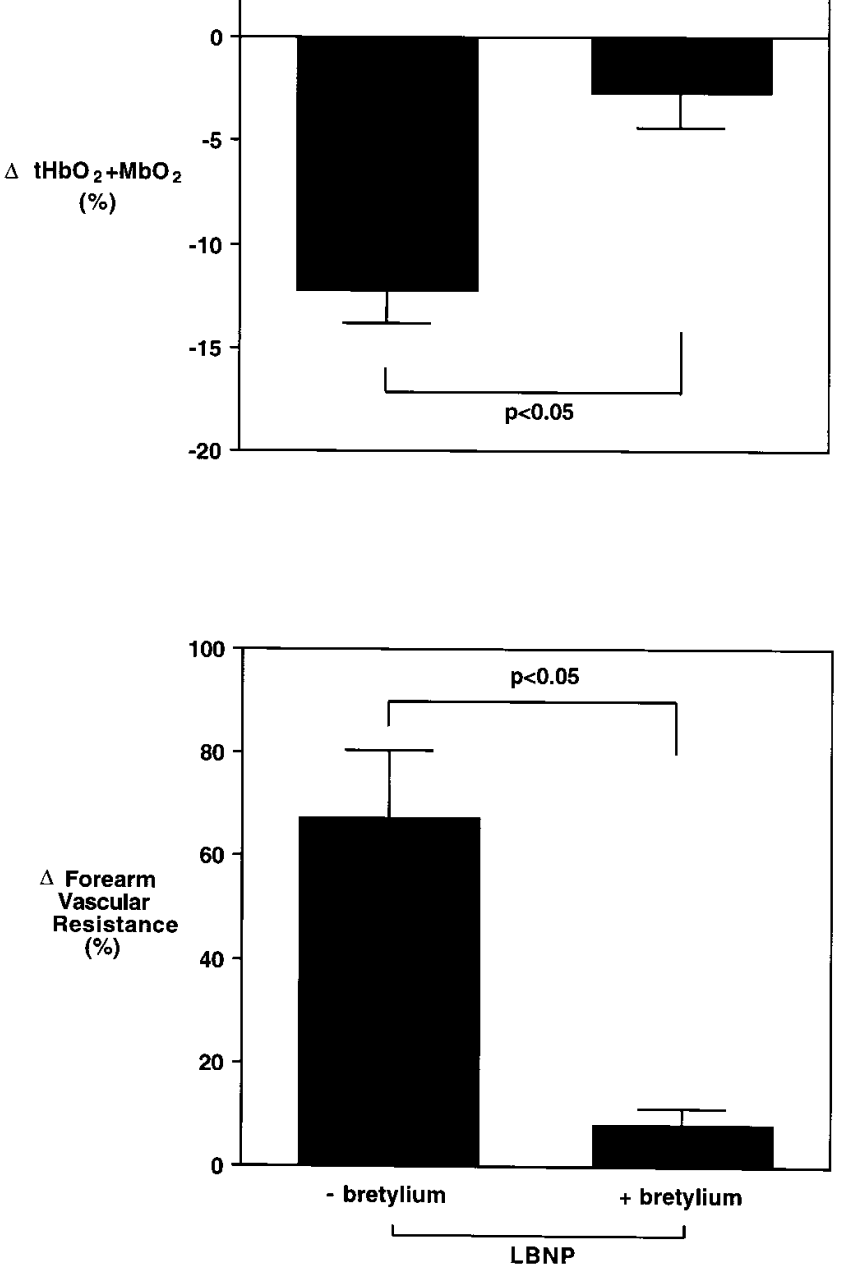

between exercise bouts. 8 min of complete circulatory arrest was performed at the end of the protocol.

Protocol 6. Effects of reflex-sympathetic activation on muscle oxygenation during reactive (as opposed to exercise-induced) hyper-
After Bretylium

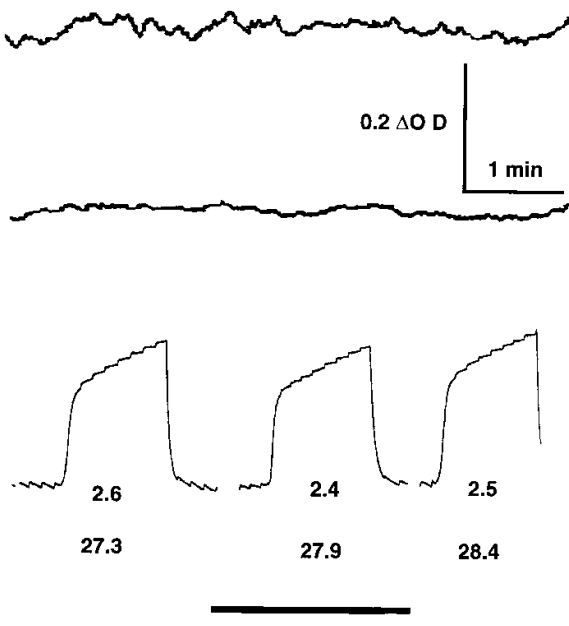

LBNP

Figure 2. (A) Segments of original near infrared optical measurements of forearm muscle oxygenation (top) and plethysmographic tracings of forearm blood flow (bottom) in response to nonhypotensive LBNP before (left) and $1 \mathrm{~h}$ after (right) forearm-sympathetic blockade with bretylium. Before bretylium, LBNP produced marked reflex vasoconstriction in the forearm as evidenced by the decrease in forearm blood flow and increase in calculated vascular resistance. This reflex vasoconstriction was accompanied by reductions in muscle oxygenation that were sympathetically mediated, because, like the decreases in forearm blood flow, they were abolished after forearm-sympathetic blockade with bretylium. $(B)$ Summary data showing the changes in forearm muscle oxygenation ( $\mathrm{tHbO}_{2}+\mathrm{MbO}_{2}$, percentage of total labile signal) (top) and calculated forearm vascular resistance (bottom) in response to LBNP before and after forearm sympathetic blockade with bretylium. Data represent mean \pm SEM, $n=4$.

emia (4 experiments performed on 4 subjects). Reactive hyperemia was used as an internal control for any nonspecific effects of exerciseinduced hyperemia on the changes in muscle oxygenation induced by a given reflex increase in muscle SNA (evoked by LBNP). 

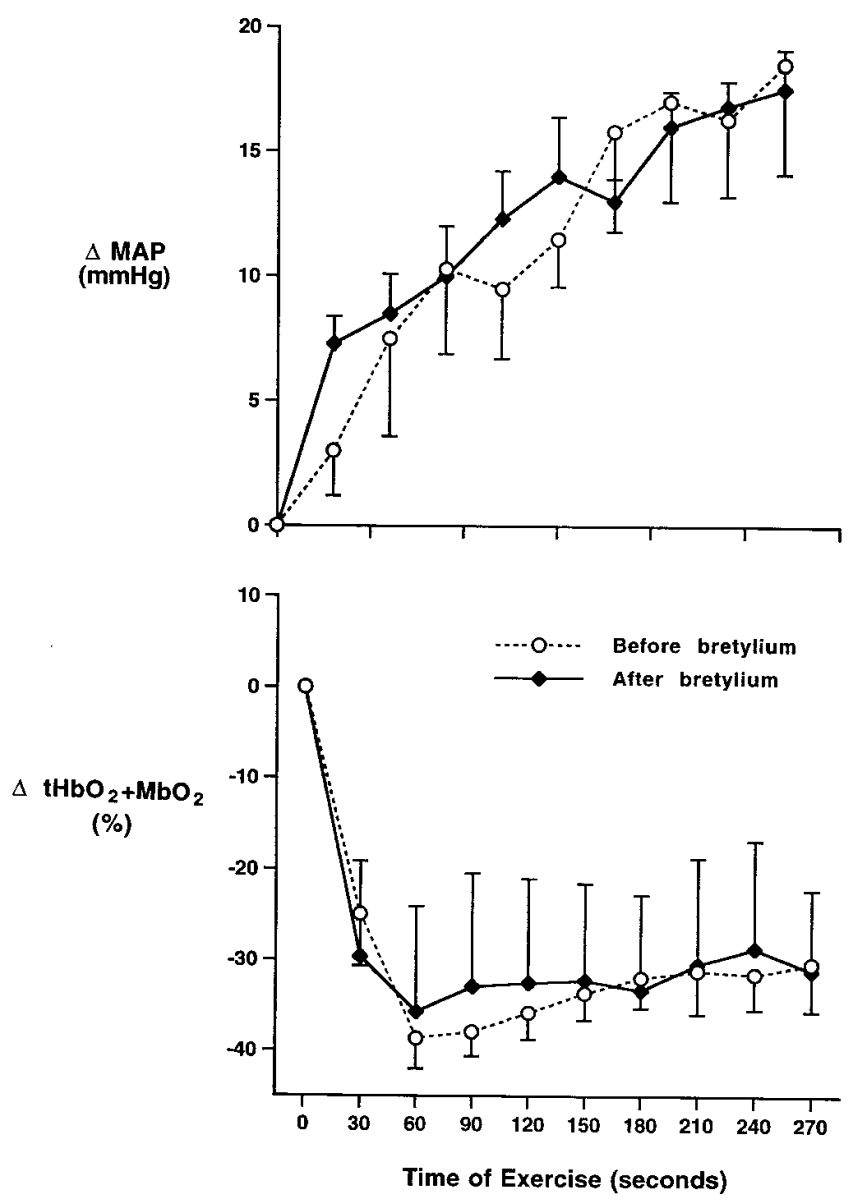

Figure 3. Summary data showing the decreases in muscle oxygenation $\left(\mathrm{tHbO}_{2}+\mathrm{MbO}_{2}\right)$ and increases in mean arterial pressure $(M A P)$ during 5 min of rhythmic handgrip at $45 \%$ maximal voluntary contraction performed before and after forearm-sympathetic blockade with bretylium. Data represent mean \pm SEM, $n=4$. During intense handgrip, the increases in mean arterial pressure and decreases in muscle oxygenation were unaffected by sympathetic blockade, indicating that local forearm-sympathetic neural activation is not an important determinant of these responses.

Blood pressure, heart rate, and near infrared signals were measured in response to LBNP ( $2 \mathrm{~min}$ at $-20 \mathrm{mmHg}$ applied at baseline, and $30 \mathrm{sec}$ after release of a complete ischemia (pneumatic cuff at 280 $\mathrm{mmHg}$ for $8 \mathrm{~min}$ ) of the resting forearm.

\section{Data analysis}

Statistical analysis was performed using repeated measures analysis of variance with Dunnett's post hoc test to detect values that were different from baseline values. Responses to handgrip before and after bretylium were compared using two-factor analysis of variance for repeated measures. Single comparisons were performed using a $t$ test for paired or unpaired comparisons. $P<0.05$ was considered significant. Data are expressed as mean \pm SEM.

\section{Results}

During high intensity rhythmic handgrip, decreases in muscle oxygenation are temporally dissociated from metaboreflex-mediated increases in muscle sympathetic nerve activity (Protocol 1, Fig. 1, and Table I). During the 1st min of rhythmic handgrip at $45 \%$ MVC, both cyt $a, a_{3}$ and $\mathrm{tHbO}_{2}+\mathrm{MbO}_{2}$ decreased rap- idly to new steady state levels, but muscle SNA remained unchanged from baseline. During the subsequent 4 min of the exercise, no additional changes in muscle oxygenation were observed even though muscle SNA increased progressively.

Reflex sympathetic vasoconstriction evokes a decrease in the oxygenation of resting forearm muscle (Protocol 2, Fig. 2). Nonhypotensive LBNP evoked reflex decreases in forearm blood flow and increases in forearm vascular resistance that were accompanied by decreases in muscle oxygenation. These decreases in muscle oxygenation indeed were sympathetically mediated because, like the decreases in forearm blood flow, they were abolished by regional sympathetic blockade with bretylium.

During high intensity rhythmic handgrip, contraction-induced decreases in muscle oxygenation are unaffected by local blockade of sympathetic neurotransmission in the forearm (Protocol 3, Fig. 3). Decreases in muscle oxygenation and increases in systemic blood pressure (muscle perfusion pressure) were indistinguishable during high intensity handgrip before and after regional sympathetic blockade of the exercising arm, demonstrating that forearm-sympathetic neural activation was not an important determinant of oxygenation in the contracting muscle. Sympathetic blockade was highly effective since it abolished optical responses at rest to LBNP at $-20 \mathrm{mmHg}$ $\left(\Delta \mathrm{tHbO}_{2}+\mathrm{MbO}_{2}:-13 \pm 3\right.$ vs $0.1 \pm 1.2 \%$ total labile signal before vs after bretylium, $P<0.05)$ and $-40 \mathrm{mmHg}\left(\Delta \mathrm{tHbO}_{2}+\right.$ $\mathrm{MbO}_{2}:-16 \pm 2$ vs $-1 \pm 2 \%$ of the total labile signal before vs. after bretylium, $P<0.05)$. Corresponding changes were observed in the cyt $a, a_{3}$ and $\mathrm{tHb}+\mathrm{Mb}$ signals.

Sympathetically mediated decreases in muscle oxygenation are eliminated when the forearm muscle is exercised, providing direct evidence for functional sympatholysis in human skeletal muscle. (Protocol 4, Figs. 4 and 5, and Table II). The reflex increase in muscle SNA induced by LBNP was identical when LBNP was applied before, during, or after performance of rhythmic handgrip at 20 or $33 \%$ MVC. The magnitude of this response, a fourfold increase in SNA over baseline, was at least as great as (and actually greater than) that produced by metaboreflex activation during handgrip at $45 \%$ MVC. This reflex increase in muscle SNA, which produced reproducible decreases in muscle oxygenation both before and after handgrip, had no detectable effect on muscle oxygenation when the same muscle was exercised during rhythmic handgrip at either 20 or $33 \%$ MVC. Thus, handgrip at 20 and $33 \%$ MVC produced steady state decreases in cyt $a, a_{3}(-32 \pm 4$ and $-45 \pm 5 \%$ of the total labile signal, respectively) and $\mathrm{tHbO}_{2}+\mathrm{MbO}_{2}$ ( $-33 \pm 5$ and $-45 \pm 7 \%$ of the total labile signal), but when the LBNP was superimposed during rhythmic handgrip, no additional decrement in these signals was observed.

In contrast, the decrease in muscle oxygenation elicited by brief mechanical occlusion of the forearm circulation was preserved and even augmented during handgrip. When the vascular occlusion was applied to the resting forearm, $\mathrm{tHbO}_{2}+\mathrm{MbO}_{2}$ decreased by $13 \pm 2 \%$ of the total labile signal $(P<0.05)$. During rhythmic handgrip at $33 \% \mathrm{MVC}$ alone, $\mathrm{tHbO}_{2}+\mathrm{MbO}_{2}$ decreased by $32 \pm 6 \%$ of the total labile signal $(P<0.05$ vs baseline), to a new steady state level. Most importantly, when the vascular occlusion was applied to the exercising forearm, $\mathrm{tHbO}_{2}+\mathrm{MbO}_{2}$ decreased by an additional $39 \pm 6 \%$ of the total labile signal $(P<0.05$ vs response at rest). Corresponding changes were observed in the cyt $a, a_{3}$ and $\mathrm{tHb}+\mathrm{Mb}$ signals.

The observed exercise-induced impairment in sympathetically mediated decreases in muscle oxygenation (i.e., func- 


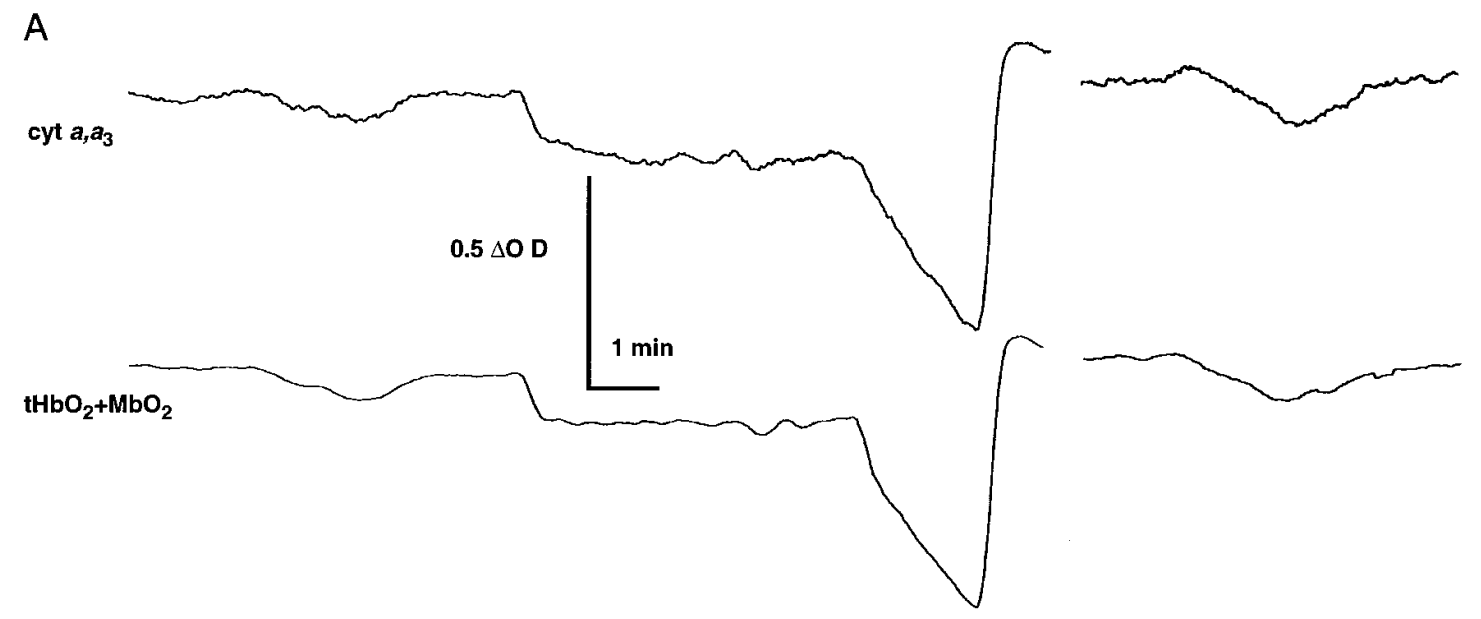

SNA
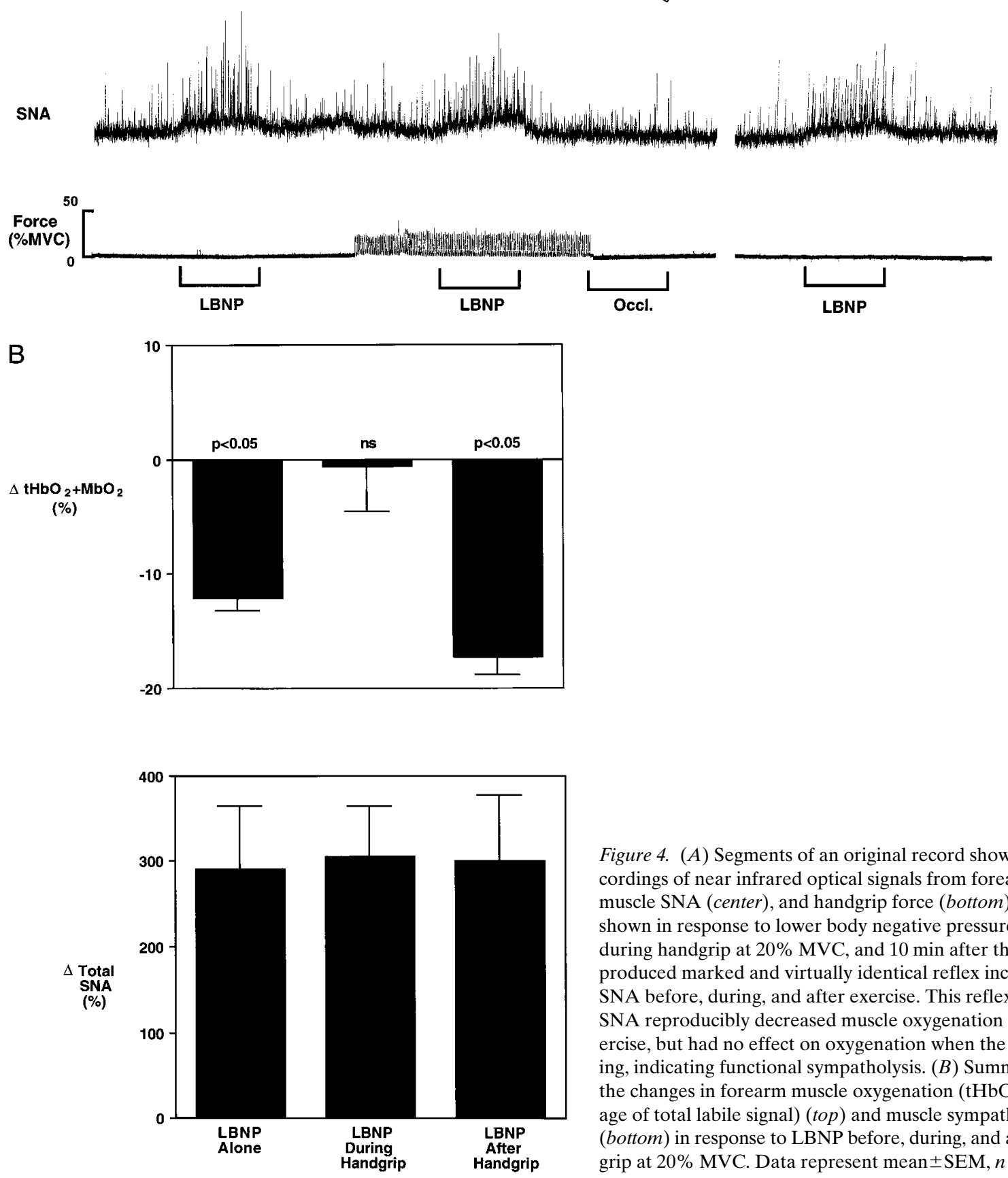

Figure 4. (A) Segments of an original record showing simultaneous recordings of near infrared optical signals from forearm muscle (top), muscle SNA (center), and handgrip force (bottom). Measurements are shown in response to lower body negative pressure $(L B N P)$ at rest, during handgrip at $20 \% \mathrm{MVC}$, and $10 \mathrm{~min}$ after the exercise. LBNP produced marked and virtually identical reflex increases in muscle SNA before, during, and after exercise. This reflex increase in muscle SNA reproducibly decreased muscle oxygenation before and after exercise, but had no effect on oxygenation when the muscle was exercising, indicating functional sympatholysis. $(B)$ Summary data showing the changes in forearm muscle oxygenation $\left(\mathrm{tHbO}_{2}+\mathrm{MbO}_{2}\right.$, percentage of total labile signal) (top) and muscle sympathetic nerve activity (bottom) in response to LBNP before, during, and after rhythmic handgrip at $20 \%$ MVC. Data represent mean \pm SEM, $n=10$. 


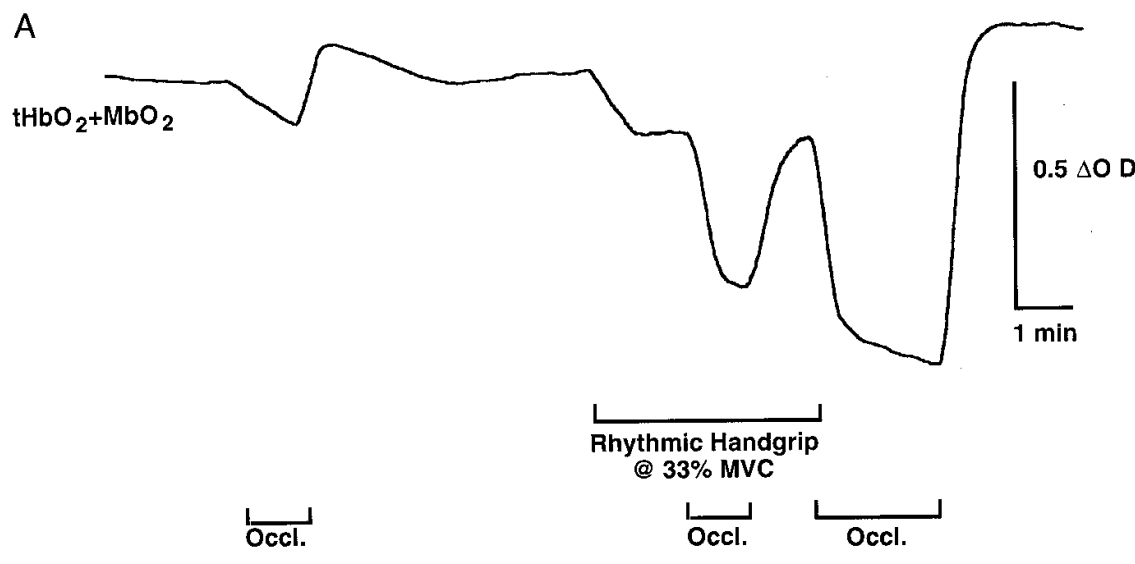

Figure 5. (A) Original near infrared optical recordings of forearm muscle $\mathrm{tHbO}_{2}+\mathrm{MbO}_{2}$ in response to brief $(1 \mathrm{~min})$ periods of forearm vascular occlusion $(\mathrm{Occl}$.) performed at rest and during rhythmic handgrip at 33\% MVC. After handgrip, forearm vascular occlusion was maintained for $2 \mathrm{~min}$. At rest, $1 \mathrm{~min}$ of vascular occlusion produced a decrease in muscle oxygenation comparable to that normally produced by 2 min of LBNP. When the muscle was exercised, vascular occlusion produced a much greater decrease in muscle oxygenation, suggesting that the sensitivity of the method to detect decreases in oxygenation (e.g., from reflex-sympathetic vasoconstriction), if present, would be at least as great in exercising as in resting muscle. $(B)$ Summary data showing changes in muscle oxygenation $\left(\mathrm{tHbO}_{2}+\mathrm{MbO}_{2}\right.$, percentage of total labile signal) in response to lower body negative pressure and brief vascular occlusion at rest and during rhythmic handgrip at $33 \%$ MVC. Responses to LBNP at rest and during rhythmic handgrip at $33 \% \mathrm{MVC}$ are reproduced from protocol 3 for comparison. Data represents mean $\pm \mathrm{SEM}, n=5$ for vascular occlusion data, $n=6$ for LBNP data.

tional sympatholysis) is localized to muscle that is being exercised. Thus, the same LBNP-induced increase in muscle SNA evoked equivalent decreases in the oxygenation of a resting forearm muscle regardless of whether the contralateral forearm was resting or exercising (rhythmic handgrip at 33\% MVC): $\Delta \mathrm{tHbO}_{2}+\mathrm{MbO}_{2}:-12 \pm 2 \%$ vs $-11 \pm 1 \%$ of the total labile signal ( $P=\mathrm{NS}$, rest vs contralateral forearm exercise).

$10-20 \%$ MVC is the minimum level of rhythmic handgrip needed to detect functional sympatholysis by near infrared spectroscopy (Protocol 5, Fig. 6). Handgrip alone at 5, 10, and 20\% MVC produced graded decreases in muscle oxygenation that reached steady state within the first $2 \mathrm{~min}$ of exercise: $\Delta \mathrm{tHbO}_{2}+\mathrm{MbO}_{2}:-8 \pm 2,-13 \pm 5$, and $-40 \pm 4 \%$ of the total labile signal, respectively, with corresponding changes in the cyt $a, a_{3}$ and $\mathrm{tHb}+\mathrm{Mb}$ signals. Reflex increases in muscle SNA induced by LBNP produced similar decreases in the oxygenation of forearm muscle when the muscle was at rest or when it was exercised during rhythmic handgrip at 5 or $10 \%$ MVC. In contrast, this sympathetically mediated decrease in muscle oxygenation was abolished during rhythmic handgrip at $20 \%$ MVC.

Sympathetically mediated decreases in forearm muscle oxygenation, although abolished during the hyperemia that accom-

Table II. Responses to LBNP before, during, and after Rhythmic Handgrip at 20 and 33\% MVC

\begin{tabular}{|c|c|c|c|c|c|c|}
\hline & \multicolumn{3}{|c|}{ Rhythmic handgrip at $20 \%$ MVC } & \multicolumn{3}{|c|}{ Rhythmic handgrip at $33 \% \mathrm{MVC}$} \\
\hline & Before & During & After & Before & During & After \\
\hline \multicolumn{7}{|l|}{$\Delta$ Mean arterial } \\
\hline pressure $(\mathrm{mmHg})$ & $4 \pm 1$ & $4 \pm 1$ & $1 \pm 1$ & $1 \pm 2$ & $7 \pm 2 *$ & $-1 \pm 2$ \\
\hline \multicolumn{7}{|l|}{$\Delta$ Muscle SNA } \\
\hline (percentage total activity) & $292 \pm 72 *$ & $305 \pm 59 *$ & $300 \pm 78 *$ & $333 \pm 119 *$ & $266 \pm 70^{*}$ & $321 \pm 27 *$ \\
\hline \multicolumn{7}{|l|}{$\Delta$ cyt $a, a_{3}$} \\
\hline (percentage TLS) & $-12 \pm 2 *$ & $-2 \pm 4$ & $-20 \pm 2 *$ & $-17 \pm 2 *$ & $-1 \pm 4$ & $-17 \pm 4 *$ \\
\hline \multicolumn{7}{|l|}{$\Delta \mathrm{tHbO}_{2}+\mathrm{MbO}_{2}$} \\
\hline (percentage TLS) & $-12 \pm 1 *$ & $-1 \pm 4$ & $-17 \pm 2 *$ & $-16 \pm 2 *$ & $4 \pm 4$ & $-18 \pm 3^{*}$ \\
\hline \multicolumn{7}{|l|}{$\Delta \mathrm{tHb}+\mathrm{Mb}$} \\
\hline (percentage TLS) & $7 \pm 1 *$ & $-1 \pm 4$ & $10 \pm 2 *$ & $11 \pm 2 *$ & $2 \pm 6$ & $13 \pm 3 *$ \\
\hline
\end{tabular}

Data shown as mean \pm SEM from 10 subjects $\left(20 \%\right.$ MVC) and 6 subjects $\left(33 \%\right.$ MVC); ${ }^{*}$ significant responses $(P<0.05)$. 


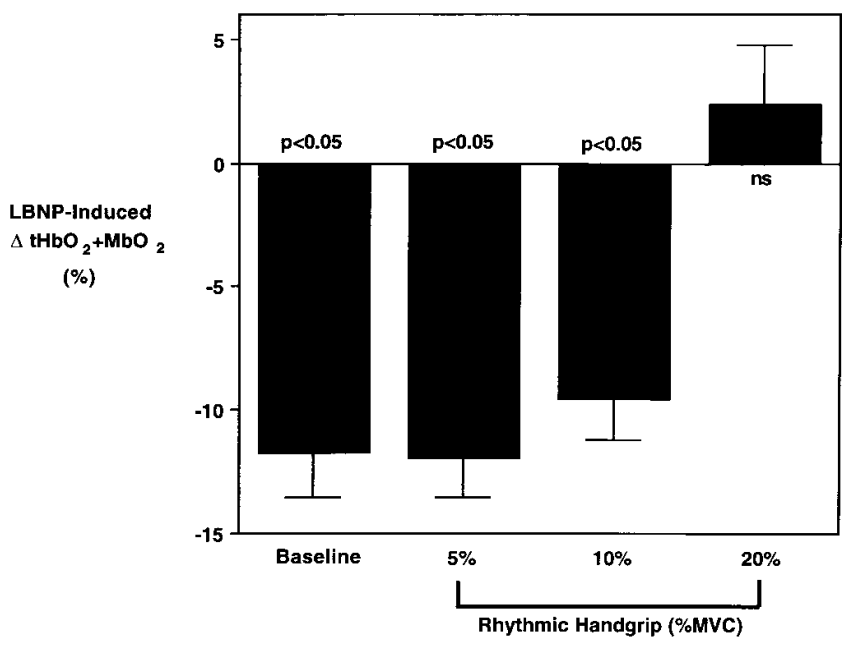

Figure 6. Summary data showing the effect of LBNP-induced reflex-sympathetic activation on forearm muscle oxygenation $\left(t \mathrm{HbO}_{2}+\mathrm{MbO}_{2}\right)$ at baseline and during graded rhythmic handgrip at 5,10 , and $20 \%$ MVC. Data represent mean \pm SEM, $n=4$. From these data, the threshold intensity of handgrip required to produce functional sympatholysis is between 10 and $20 \%$ MVC.

panies mild forearm exercise, are well preserved during maximal reactive hyperemia after ischemia of the resting forearm (Protocol 6, Fig. 7). Sympathetically mediated decreases in forearm muscle oxygenation elicited by LBNP were comparable at baseline and during peak reactive hyperemia after 8 min of forearm ischemia.

\section{Discussion}

Contraction-induced metabolic inhibition of sympathetic vasoconstriction (i.e., functional sympatholysis) has been demonstrated recently in anesthetized animal preparations $(33,44)$.
We now have provided new evidence for functional sympatholysis in conscious humans using near infrared spectroscopy. Reflex sympathetic activation, which consistently decreased oxygenation in resting forearm muscles, had no effect on oxygenation when the muscles were exercised. We also found that the threshold intensity of contraction required to evoke functional sympatholysis is unexpectedly mild, 10-20\% MVC. Because this threshold intensity is far below that required for muscle metaboreflex activation (i.e., 33-45\% MVC), the data suggest that the two processes are governed by different mechanisms.

By using several different experimental approaches, we repeatedly dissociated decreased oxygenation from increased SNA in contracting skeletal muscle. During high intensity rhythmic handgrip (45\% MVC), the contraction-induced decrease in muscle oxygenation reached a nadir before the onset of reflex-sympathetic activation and it did not decrease further despite the subsequent slow and progressive increase in muscle SNA. This temporal dissociation provided the first clue to the existence of functional sympatholysis, since the decreases in muscle oxygenation were far from maximal and therefore would not have precluded detection of an additional decrement in muscle oxygenation when muscle SNA was increasing during the latter part of the exercise. We further dissociated muscle oxygenation from muscle SNA during high intensity handgrip by showing that the contraction-induced deoxygenation was unaffected by eliminating sympathetic neurotransmission in the exercising muscles. During milder levels of rhythmic handgrip (20-33\% MVC), experimentally induced (i.e., baroreflex-mediated) increases in muscle SNA had no effect on oxygenation in the exercising muscles, but they decreased oxygenation in the resting muscles. From these observations, we conclude that reflex-sympathetic activation in contracting skeletal muscle is not an important determinant of muscle oxygenation.

That these observations demonstrate functional sympatholysis is predicated on the assumption that the sensitivity
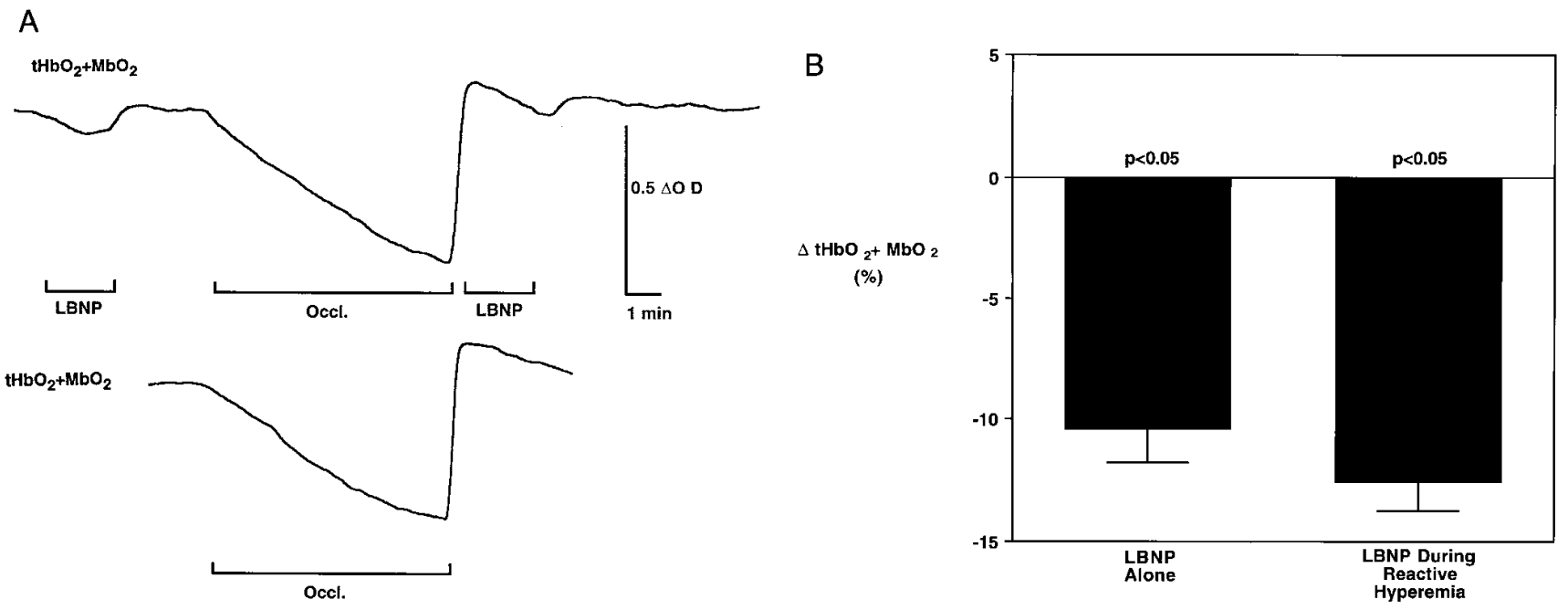

Figure 7. (A) Original near infrared optical recordings of forearm muscle $t \mathrm{HbO}_{2}+\mathrm{MbO}_{2}$ in response to $\mathrm{LBNP}$ (top) at baseline and during peak reactive hyperemia after $8 \mathrm{~min}$ of forearm ischemia $(\mathrm{Occl}$.). The response to forearm ischemia alone (bottom) is shown for comparison. Reflex sympathetic activation with LBNP evoked a comparable decrease in muscle oxygenation at rest and during reactive hyperemia after forearm vascular occlusion. $(B)$ Summary data showing forearm muscle oxygenation ( $\mathrm{tHbO}_{2}+\mathrm{MbO}_{2}$, percentage of total labile signal) in response to LBNP before and during reactive hyperemia after $8 \mathrm{~min}$ of ischemia. Data represent mean $\pm \mathrm{SEM}, n=4$. 
of near infrared spectroscopy to detect decreased oxygen delivery is preserved when muscle blood flow and oxygen consumption are increased as during exercise. Because near infrared indices of muscle oxygenation reflect the balance between oxygen delivery and demand, a given decrease in oxygen delivery would be expected to produce, if anything, a larger decrease in muscle oxygenation during contraction (high demand) than at rest (low demand). Indeed, Mancini et al. (61) demonstrated that brachial artery infusion of vasoconstrictor agents produced decreases in forearm muscle oxygenation that were greater during mild rhythmic forearm exercise than at rest. We demonstrated that elimination of oxygen delivery with forearm circulatory arrest produced a larger decrease in muscle oxygenation when applied during handgrip than at rest. We also demonstrated that sympathetically mediated reflex decreases in muscle oxygenation were well preserved during reactive hyperemia; i.e., during increases in blood flow and hemoglobin in the muscle vessels of similar magnitude to those occurring during exercise.

The present experiments demonstrate that functional sympatholysis is localized to the exercising muscles, which is consistent with our previous findings in rats (33). Because sympathetically mediated decreases in muscle oxygenation were preserved in resting forearm muscles during contralateral forearm muscle contraction, we conclude that functional sympatholysis is mediated by some local metabolic event(s) and not by some blood-borne factor.

The demonstration of functional sympatholysis by near infrared spectroscopy by no means refutes previous hemodynamic data indicating sympathetic vasoconstriction in exercising human skeletal muscle (20-28). In this regard, near infrared spectroscopy provides several important advantages over traditional hemodynamic approaches to study metabolic modulation of sympathetic vasoconstriction in exercising humans. First, the technique provides continuous measurement of oxygen availability at the level of microcirculation, the part of the vascular tree most accessible to metabolic products of contraction $(48-50,61)$. In contrast, measurements of wholelimb blood flow with techniques such as venous occlusion plethysmography $(21-24,26,28)$ or indicator dilution $(20,27)$ reflect the vasomotor tone of mainly resistance vessels, which are not so accessible to metabolites produced in the muscle interstitium. We speculate that during exercise sympathetic vasoconstriction in upstream resistance vessels contributes to blood pressure regulation while sympatholysis in mainly the downstream nutrient vessels optimizes muscle perfusion $(33,34,42$, $68)$. Second, the optrode placement is sufficiently stable to acquire measurements even during high intensity rhythmic contraction. This is a major advantage over techniques (e.g., venous occlusion plethysmography) that permit blood flow measurements only after the cessation of exercise, since many of the neurocirculatory adjustments during exercise return to baseline within seconds after the cessation of exercise $(6,69)$.

Third, and most importantly, the spatial resolution of near infrared spectroscopy is sufficient to reflect changes in oxygenation in the truly active (rather than adjacent inactive) small muscle groups of the forearm. This is a major advantage for studying sympatholysis, a phenomenon localized to only active muscle. In contrast, venous effluent blood is an admixture of blood draining active and adjacent nonactive muscle groups, thereby reducing the sensitivity of venous oxygen saturation to detect changes in oxygenation in the active muscle $(25,26)$. We suggest that sympatholysis in the active muscle coupled with sympathetic vasoconstriction in adjacent inactive muscle optimally redistributes intramuscular blood flow to the most active fibers $(33,34,42,70)$.

The present study does not determine whether functional sympatholysis is mediated primarily via a pre- or postjunctional site of action. In ex vivo vascular preparations, prejunctional inhibition of norepinephrine release from peripheral sympathetic nerve terminals has been demonstrated by cotransmitters such as neuropeptide Y (71) or substances such as nitric oxide, adenosine, or $\mathrm{K}^{+}(72-75)$, whose intramuscular concentration may increase during exercise. In humans, however, norepinephrine spillover from exercising skeletal muscle clearly is not inhibited during contraction; during unilateral quadriceps exercise, norepinephrine spillover is even greater from the exercising than from the nonexercising muscle (76). In previous animal studies, functional sympatholysis was evident with either sympathetic nerve stimulation or direct application of exogenous alpha-adrenergic agonists, indicating a postjunctional site of action (33).

Although the local metabolic events mediating functional sympatholysis remain to be defined, we performed several additional sets of experiments that provide some important clues regarding potential underlying mechanisms. Sympathetically mediated decreases in muscle oxygenation, while abolished during contraction-induced hyperemia, were well preserved during reactive hyperemia. This observation demonstrates that functional sympatholysis is specific for exercise and is not merely a nonspecific effect of muscle hyperemia overwhelming sympathetic vasoconstrictor drive. This observation further suggests that the various local vasodilator metabolites that have been implicated in mediating reactive hyperemia (adenosine, inorganic phosphate, histamine, and prostaglandins, reference 73) all are unlikely candidates to be primary mediators of functional sympatholysis.

Hydrogen ion $\left(\mathrm{H}^{+}\right)$initially was an attractive candidate to mediate functional sympatholysis in humans for several reasons. Our previous animal experiments (33) advanced the hypothesis that glycolytic production of $\mathrm{H}^{+}$mediates functional sympatholysis in rat hindlimb muscle. This is consistent with microcirculatory studies demonstrating that adrenergic vasoconstriction is impaired by experimental acidosis $(39,42)$. Previous human microneurographic studies $(8,11,13)$ advanced the hypothesis that glycolytic production of $\mathrm{H}^{+}$in exercising skeletal muscle mediates activation of the muscle metaboreflex. This is consistent with single-fiber recordings of muscle metaboreceptor afferent discharge in cats, demonstrating that lactic acid is one of the most potent stimuli to these afferents (5). Taken together, this previous work led us to hypothesize that glycolytic production of $\mathrm{H}^{+}$is involved in the coordinate regulation of reflex-sympathetic activation and mitochondrial oxygenation in contracting skeletal muscle. Based on the present experiments however, $\mathrm{H}^{+}$is unlikely to be the primary mediator of functional sympatholysis in humans, because sympathetically mediated decreases in muscle oxygenation were eliminated completely during even mild rhythmic handgrip (as low as $20 \%$ MVC), which does not cause cellular acidification and does not activate the muscle metaboreflex (7-9). This was a surprising finding because our previous rat experiments (33) suggested that near maximal contractions were required to elicit functional sympatholysis. The precise explanation for this apparent difference in the conclusions derived from these different models is unknown, 
but may be related to several factors including species, general anesthesia, mode of contraction (electrically evoked vs voluntary), or sensitivity for the detection of functional sympatholysis using Doppler velocimetry vs near infrared spectroscopy.

A salient finding of the present study is that even mild levels of exercise (10-20\% MVC) are accompanied by sizable decreases in muscle oxygenation, raising the possibility that skeletal muscle hypoxia per se is a primary determinant of functional sympatholysis. This is an attractive hypothesis because tissue hypoxia has been shown to impair sympathetic vasoconstriction in rodent and feline skeletal muscle microvessels both $(a)$ directly, by interfering with oxygen-sensitive adrenergic signal transduction pathways (such as those involving ATP-sensitive $\mathrm{K}^{+}$channels) in vascular smooth muscle, and (b) indirectly, by causing release of vasodilator metabolites from the surrounding skeletal muscle tissue in which the vessels are embedded $(41,45)$. The relative importance of such cellular mechanisms in mediating functional sympatholysis is beyond the scope of the present study.

Regardless of the precise underlying mechanisms involved, the present study demonstrates in humans that sympathetic neural control of skeletal muscle oxygenation normally is very sensitive to modulation by local metabolic events in the contracting muscles. Based on our experimental findings in healthy humans, we speculate that, in the clinical setting, impaired functional sympatholysis may contribute to decreased exercise capacity in pathophysiologic conditions such as heart failure or renal failure, which are characterized by profound alterations in both skeletal muscle metabolism and sympathetic nerve activity.

\section{Acknowledgments}

We thank Dr. Claude A. Piantadosi for generously providing the near infrared technology, and Mark Hall for superb technical assistance. We thank Dr. Jere H. Mitchell for his critical review of our work.

Dr. Hansen is the recipient of the National Institutes of Health's (NIH's) Fogarty International Research Fellowship (NIH-1-F05TW04949-01) and was supported by grants from the Danish Heart Foundation, the Simonsen \& Weel Foundation, and the Danish Research Academy. Dr. Thomas was supported by an NIH training grant (T32-HL-07360). Dr. Parsons is the recipient of a Clinician-Scientist Award from the American Heart Association and Genentech. Dr. Victor is an Established Investigator of the American Heart Association. This research was supported by a grant to Dr. Victor from the NIH (PO1-HL-06296) and by American Heart Association Texas Affiliate grant-in-aid (96G-064) to Dr. Thomas.

\section{References}

1. Volkmann, A.W. 1841. Bewegungen des atmens und schluckens mit besonderer berucksichtigung neurologischer streitfragen. Arch. Anat. Physiol. 1: 332-360.

2. Mitchell, J.H., M.P. Kaufman, and G.A. Iwamoto. 1983. The exercise pressor reflex: its cardiovascular effects, afferent mechanisms, and central pathways. Annu. Rev. Physiol. 45:229-242.

3. Mense, S., and M. Stahnke. 1983. Responses in muscle afferent fibres of slow conduction velocity to contractions and ischemia in the cat. J. Physiol. 342: 383-397.

4. Kaufman, M.P., J.C. Longhurst, K.J. Rybicki, J.H. Wallach, and J.H. Mitchell. 1983. Effects of static muscular contraction on impulse activity of groups III and IV afferents in cats. J. Appl. Physiol. 55:105-112.

5. Rotto, D.M., C.L. Stebbins, and M.P. Kaufman. 1989. Reflex cardiovascular and ventilatory responses to increasing $\mathrm{H}^{+}$activity in cat hindlimb muscle. J. Appl. Physiol. 67:256-263.

6. Mark, A.L., R.G. Victor, C. Nerhed, and B.G. Wallin. 1985. Microneurographic studies of the mechanisms of sympathetic nerve responses to static ex- ercise in humans. Circ. Res. 57:461-469.

7. Victor, R.G., D.R. Seals, and A.L. Mark. 1987. Differential control of heart rate and sympathetic nerve activity during dynamic exercise. Insight from intraneural recordings in humans. J. Clin. Invest. 79:508-516.

8. Victor, R.G., L.A. Bertocci, S.L. Pryor, and R.L. Nunnally. 1988. Sympathetic nerve discharge is coupled to muscle cell $\mathrm{pH}$ during exercise in humans. $J$. Clin. Invest. 82:1301-1305.

9. Victor, R.G., and D.R. Seals. 1989. Reflex stimulation of sympathetic outflow during rhythmic exercise in humans. Am. J. Physiol. 257:2017-2024.

10. Seals, D. 1989. Sympathetic neural discharge and vascular resistance during exercise in humans. J. Appl. Physiol. 66:2472-2478.

11. Pryor, S.L., S.F. Lewis, R.G. Haller, L.A. Bertocci, and R.G. Victor 1990. Impairment of sympathetic activation during static exercise in patients with muscle phosphorylase deficiency (McArdle's disease). J. Clin. Invest. 85: 1444-1449.

12. Scherrer, U., S.L. Pryor, L.A. Bertocci, and R.G. Victor. 1990. Arterial baroreflex buffering of sympathetic activation during exercise-induced elevations in arterial pressure. J. Clin. Invest. 86:1855-1861.

13. Ettinger, S., K. Gray, S. Whisler, and L. Sinoway. 1991. Dichloroacetate reduces sympathetic nerve responses to static exercise. Am. J. Physiol. 261 $1653-1658$.

14. Sinoway, L.I., R.F. Rea, T.J. Mosher, M.B. Smith, and A.L. Mark. 1992. Hydrogen ion concentration is not the sole determinant of muscle metaboreceptor responses in humans. J. Clin. Invest. 89:1875-1884.

15. Costa, F., and I. Biaggioni. 1994. Role of adenosine in the sympathetic activation produced by isometric exercise in humans. J. Clin. Invest. 93:1654 1660.

16. Hansen, J., G.D. Thomas, T.N. Jacobsen, and R.G. Victor. 1994. Muscle metaboreflex triggers parallel sympathetic activation in exercising and resting human skeletal muscle. Am. J. Physiol. 266:2508-2514.

17. Thompson, L.P., and D.E. Mohrman. 1983. Blood flow and oxygen consumption in skeletal muscle during sympathetic stimulation. Am. J. Physiol. 245:66-71.

18. Peterson, D.F., R.B. Armstrong, and H.M. Laughlin. 1988. Sympathetic neural influences on muscle blood flow in rats during submaximal exercise. $J$ Appl. Physiol. 65:434-440.

19. O'Leary, D.S., L.B. Rowell, and A.M. Scher. 1991. Baroreflex-induced vasoconstriction in active skeletal muscle of conscious dogs. Am. J. Physiol. 260:37-41.

20. Secher, N.H., J.P. Clausen, K. Klausen, I. Noer, and J. Trap-Jensen. 1977. Central and regional circulatory effects of adding arm exercise to leg exercise. Acta Physiol. Scand. 100:288-297.

21. Williams, C.A., J.G. Mudd, and A. R. Lind. 1985. Sympathetic control of the forearm blood flow in man during brief isometric contractions. Eur. J. Appl. Physiol. 54:156-162.

22. Sinoway, L.I., J.S. Wilson, R. Zelis, J. Shenberger, D.P. McLaughlin, D.L. Morris, and F.P. Day. 1988. Sympathetic tone affects human limb vascular resistance during a maximal metabolic stimulus. Am. J. Physiol. 255(4 Pt 2): H937-946.

23. Sinoway, L., and S. Prophet. 1990. Skeletal muscle metaboreceptor stimulation opposes peak metabolic vasodilation in humans. Circ. Res. 66:15761584 .

24. Joyner, M.J., R.L. Lennon, D.J. Wedel, S.H. Rose, and J.T. Shepherd. 1990. Blood flow to contracting human muscles: influence of increased sympathetic activity. J. Appl. Physiol. 68:1453-1457.

25. Joyner, M.J. 1991. Does the pressor response to ischemic exercise improve blood flow to contracting muscles in humans? J. Appl. Physiol. 71:14961501.

26. Joyner, M.J., L.A. Nauss, M.A. Warner, and D.O. Warner. 1992. Sympathetic modulation of blood flow and $\mathrm{O}_{2}$ uptake in rhythmically contracting human forearm muscles. Am. J. Physiol. 263:1078-1083.

27. Richter, E.A., B. Kiens, M. Hargreaves, and M. Kjær. 1992. Effect of arm-cranking on leg blood flow and noradrenaline spillover during leg exercise in man. Acta Physiol. Scand. 144:9-14.

28. Saito, M., A. Kagaya, F. Ogita, and M. Shinohara. 1992. Changes in muscle sympathetic nerve activity and calf blood flow during combined leg and forearm exercise. Acta Physiol. Scand. 146:449-456.

29. Rowell, L.B. 1993. Human Cardiovascular Control. Oxford University Press, New York. 471-475.

30. Remensnyder, J.P., J.H. Mitchell, and S.J. Sarnoff. 1962. Functional sympatholysis during muscular activity. Circ. Res. 11:370-380.

31. Kjellmer, I. 1965. On the competition between metabolic vasodilatation and neurogenic vasoconstriction in skeletal muscle. Acta Physiol. Scand. 63: 450-459.

32. Donald, D.E., D.J. Rowlands, and D.A. Ferguson. 1970. Similarity of blood flow in the normal and the sympathectomized dog hind limb during graded exercise. Circ. Res. 26:185-199.

33. Thomas, G.D., J. Hansen, and R.G. Victor. 1994. Inhibition of alpha 2 -adrenergic vasoconstriction during contraction of glycolytic, not oxidative, rat hindlimb muscle. Am. J. Physiol. 266:920-929.

34. Strandell, T., and J.T. Shepherd. 1967. The effect in humans of increased sympathetic activity on the blood flow to active muscles. Acta Med. Scand. 
Suppl. 472:146-167.

35. Hartling, O.J., and J. Trap-Jensen. 1983. Hemodynamic and metabolic effects of alpha-adrenoceptor blockade with phentolamine at rest and during forearm exercise. Clin. Sci. (Lond.). 65:247-253.

36. Savard, G.K., E.A. Richter, S. Strange, B. Kiens, N.J. Christensen, and B. Saltin. 1989. Norepinephrine spillover from skeletal muscle during exercise in humans: role of muscle mass. Am. J. Physiol. 257:1812-1818.

37. Rowell, L.B., M.V. Savage, J. Chambers, and J.R. Blackmon. 1991. Cardiovascular responses to graded reductions in leg perfusion in exercising humans. Am. J. Physiol. 261:1545-1553.

38. Richardson, R.S., B. Kennedy, D.R. Knight, and P.D. Wagner. 1995. High muscle blood flows are not attenuated by recruitment of additional muscle mass. Am. J. Physiol. 269:1545-1552.

39. Medgett, I.C., P.E. Hicks, and S.Z. Langer. 1987. Effect of acidosis on alpha1 and alpha2 adrenoreceptor mediated vasoconstrictor responses in isolated arteries. Eur. J. Pharmacol. 135:443-447.

40. Boegehold, M.A., and P.C. Johnson. 1988. Response of arteriolar network of skeletal muscle to sympathetic nerve stimulation. Am. J. Physiol. 254: 919-928.

41. Boegehold, M.A., and P.C. Johnson. 1988. Periarteriolar and tissue $\mathrm{PO}_{2}$ during sympathetic escape in skeletal muscle. Am. J. Physiol. 254:929-936.

42. McGillivray-Anderson, K.M., and J.E. Faber. 1990. Effect of acidosis on contraction of microvascular smooth muscle by alpha1- and alpha2-adrenoceptors. Implications for neural and metabolic regulation. Circ. Res. 66:1643-1657.

43. McGillivray-Anderson, K.M., and J.E. Faber. 1991. Effect of reduced blood flow on alpha1- and alpha2-adrenoceptor constriction of rat skeletal muscle microvessels. Circ. Res. 69:165-173.

44. Anderson, K.M., and J.E. Faber. 1991. Differential sensitivity of arteriolar alpha 1- and alpha 2-adrenoceptor constriction to metabolic inhibition during rat skeletal muscle contraction. Circ. Res. 69:174-184.

45. Tateishi, J., and J.E. Faber. 1995. ATP-sensitive $\mathrm{K}^{+}$channels mediate alpha 2D-adrenergic receptor contraction of arteriolar smooth muscle and reversal of contraction by hypoxia. Circ. Res. 76:53-63.

46. Delius, W., K.-E. Hagbarth, A. Hongell, and B. Wallin. 1972. Manoeuvres affecting sympathetic outflow in human nerves. Acta Physiol. Scand. 84: $82-94$

47. Vallbo, Å., K.-E. Hagbarth, H. Torebjork, and B. Wallin. 1979. Somatosensory, proprioceptive, and sympathetic activity in human peripheral nerves. Physiol. Rev. 59:919-957.

48. Piantadosi, C.A. 1989. Near infrared spectroscopy: principles and application to noninvasive assessment of tissue oxygenation. J. Crit. Care. 4:308-318.

49. Piantadosi, C.A., and B.J. Comfort. 1989. Development and validation of multiwavelength algorithms for in vivo near infrared spectroscopy. In Photon Migration in Tissue. B. Chance, editor. Plenum Publishing Corp., New York. 69-82.

50. Piantadosi, C.A. 1993. Absorption spectroscopy for assessment of mitochondrial function in vivo. In Mitochondrial Dysfunction. Vol. 2. L.H. Lash and D.P. Jones, editors. Academic Press Inc., San Diego, CA. 107-126.

51. Jobsis, F.F. 1977. Noninvasive infrared monitoring of cerebral and myocardial and circulatory parameters. Science (Wash. DC). 198:1264-1267.

52. Hampson, N.B., and C.A. Piantadosi. 1988. Near infrared monitoring of human skeletal muscle oxygenation during forearm ischemia. J. Appl. Physiol. 64:2449-2457.

53. Parsons, W.J., J.C. Rembert, R.P. Bauman, J.C. Greenfield, Jr., and C.A. Piantadosi. 1990. Dynamic mechanisms of cardiac oxygenation during brief ischemia and reperfusion. Am. J. Physiol. 259:1477-1485.

54. Duhaylongsod, F.G., J.A. Griebel, D.S. Bacon, W.G. Wolfe, and C.A. Piantadosi. 1993. Effects of muscle contraction on cytochrome a,a3 redox state. J. Appl. Physiol. 75:790-797.

55. Parsons, W.J., J.C. Rembert, R.P. Bauman, F.G. Duhaylongsod, J.C. Greenfield, Jr., and C.A. Piantadosi. 1993. Myocardial oxygenation in dogs during partial and complete coronary artery occlusion. Circ. Res.. 73:458-464.

56. Piantadosi, C.A., and F.G. Duhaylongsod. 1994. Near infrared spectros- copy: in situ studies of skeletal and cardiac muscle. Adv. Exp. Med. Biol. 361: 157-161.

57. Chance, B., and W. Bank. 1995. Genetic disease of mitochondrial function evaluated by NMR and NIR spectroscopy of skeletal tissue. Biochim. Biophys. Acta. 1271:7-14.

58. Tamura, M., O. Hazeki, S. Nioka, and B. Chance. 1989. In vivo study of tissue oxygen metabolism using optical and nuclear magnetic resonance spectroscopies. Annu. Rev. Physiol. 51:813-834.

59. Piantadosi, C.A., J.A. Griebel, and N.B. Hampson. 1988. Intramitochondrial oxygen decreases in forearm muscle during venous congestion. Clin. Res. 36:37. (Abstr.)

60. Piantadosi, C.A. 1989. Behavior of the copper band of cytochrome c oxidase in rat brain during FC-43-for-blood substitution. Adv. Exp. Med. Biol. 248:81-90.

61. Mancini, D.M., L. Bolinger, H. Li, B. Kendrick, B. Chance, and J.R. Wilson. 1994. Validation of near infrared spectroscopy in humans. J. Appl. Physiol. 77:2740-2747.

62. Fleckenstein, J.L., D. Watumull, L.A. Bertocci, R.W. Parkey, and R.M. Peshock. 1992. Finger-specific flexor recruitment in humans: depiction by exercise-enhanced MRI. J. Appl. Physiol. 72:1974-1977.

63. Siggaard-Andersen, O. 1970. Venous occlusion plethysmography on the calf. Dan. Med. Bull. 17:1-68.

64. Victor, R.G., and W.N. Leimbach, Jr. 1987. Effects of lower body negative pressure on sympathetic discharge to leg muscles in humans. J. Appl. Physiol. 63:2558-2562.

65. Jacobsen, T.N., B.J. Morgan, U. Scherrer, S.F. Vissing, R.A. Lange, N. Johnson, W.S. Ring, P.S. Rahko, P. Hanson, and R.G. Victor. 1993. Relative contributions of cardiopulmonary and sinoaortic baroreflexes in causing sympathetic activation in the human skeletal muscle circulation during orthostatic stress. Circ. Res. 73:367-378.

66. Lefkowitz, R.J., B.B. Hoffman, and P. Taylor. 1990. Neurohumoral Transmission. In Goodman and Gilman's The Pharmacologic Basis of Therapeutics. A. Goodman, A. Gilman, T. W. Rall, A. S. Nies, and P. Taylor, editors Pergamon Press Inc., Tarrytown, NY. 84-122.

67. Wallin, B.G., R.G. Victor, and A.L. Mark. 1989. Sympathetic outflow to resting muscles during static handgrip and postcontraction muscle ischemia. Am. J. Physiol. 256:105-110.

68. Granger, H.J., A.H. Goodman, and D.N. Granger. 1976. Role of resistance and exchange vessels in local microvascular control of skeletal muscle oxygenation in the dog. Circ. Res. 38:379-385.

69. Victor, R.G., N.H. Secher, T. Lyson, and J.H. Mitchell. 1995. Central command increases muscle sympathetic nerve activity during intense intermittent isometric exercise in humans. Circ. Res. 76:127-131.

70. Nellis, S.H., S.F. Flaim, K.M. McCauley, and R. Zelis. 1980. alpha-Stimulation protects exercise increment in skeletal muscle oxygen consumption. Am. J. Physiol. 238:331-339.

71. Lundberg, J.M., A. Franco-Cereceda, J.S. Lacroix, and J. Pernow. 1990. Neuropeptide Y and sympathetic neurotransmission. Ann. N.Y. Acad. Sci. 611 $166-174$.

72. Vanhoutte, P.M., T.J. Verbueren, and R.C. Webb. 1981. Local modulation of adrenergic neuroeffector interaction in the blood vessel wall. Physiol. Rev. 61:151-247.

73. Shepherd, J.T. 1983. Circulation to skeletal muscle. In Peripheral Circulation and Organ Blood Flow. Vol. 3. J.T. Shepherd and F.M. Abboud, editors. American Physiological Society, Bethesda, MD. 319-370.

74. Cohen, R.A., and R.M. Weisbrod. 1988. Endothelium inhibits norepinephrine release from adrenergic nerves of rabbit carotid artery. Am. J. Phys iol. 254:871-878.

75. Burnstock, G. 1990. Local mechanisms of blood flow control by perivascular nerves and endothelium. J. Hypertens. 8(Suppl. 7):95-106.

76. Savard, G., S. Strange, B. Kiens, E.A. Richter, N.J. Christensen, and B. Saltin. 1987. Noradrenaline spillover during exercise in active versus resting skeletal muscle in man. Acta Physiol. Scand. 131:507-515. 